\title{
Thermal emission control with one-dimensional metallodielectric photonic crystals
}

\author{
Arvind Narayanaswamy and Gang Chen* \\ Department of Mechanical Engineering, Massachusetts Institute of Technology, Cambridge, Massachusetts 02139, USA
}

(Received 6 April 2004; published 2 September 2004)

\begin{abstract}
We have analyzed thermal emission from one-dimensional metallodielectric periodic structures using a Green's function technique together with the fluctuation-dissipation theorem. We show that such simple structures exhibit excellent selective emission characteristics with a controllable transition frequency in the infrared and even visible range. Such simple structures should be easy to fabricate and have applications in thermophotovoltaics and potentially in incandescent bulbs.
\end{abstract}

DOI: 10.1103/PhysRevB.70.125101

PACS number(s): 42.70.Qs, 41.20.-q, 42.25.Bs, 44.40.+a

The performance of thermophotovoltaic (TPV) energy conversion systems is greatly affected by the radiation characteristics of the thermal emitter. Only photons with energy larger than the band gap of the photovoltaic material can be converted to electrical power whereas those below the band gap add to the energy loss. Ideally, one would want an emitter with high emissivity above the band gap and low emissivity below the band gap. Recently, it has been shown that three-dimensional (3D) photonic crystals (PC) made of tungsten can tailor the emissivity of the structure appropriately, resulting in improved performance. ${ }^{1,2}$ The 3D PC, however, is an intricate structure that is difficult to fabricate and scale up. Two-dimensional periodic structures microfabricated on metallic films also show control over emissivity due to surface plasmon polariton effects. ${ }^{3,4}$ In this paper, we present a simple one-dimensional (1D) PC structure, requiring very little microfabrication, to control the emissivity. The results of our analysis indicate that this control can be easily extended from the IR to the visible. This kind of control over thermal emissivity could have implications not only for TPV conversion but also for other applications, including incandescent light bulb technology.

Propagation of electromagnetic waves through PCs has been studied extensively over the last two decades. Metallodielectric PCs (MDPCs) have been investigated for applications in the long wavelength $(>25 \mu \mathrm{m})$ regime, where metals act as near-perfect reflectors. ${ }^{5,6}$ In the near IR to UV regions, metals tend to become very dissipative and do not seem to be good candidates for PCs. Contrary to the above logic, it was shown that ultrathin films of metals like Ag or $\mathrm{Au}$ can be used to design optimal reflectors because of their large photonic band gaps. ${ }^{7-9}$ More recently, low-absorbing metallic inclusions in PCs have been used to achieve complete photonic band gaps in the near IR, which are not possible with dielectric structure alone. ${ }^{10}$ In this paper, we analyze thermal emission from ID PCs made of (1) thin silver films and (2) thin tungsten films. Since silver is a low-loss material, analysis of emission from PCs made of silver yields great insight into the effects of the photonic structure on the resulting radiation. We then analyze emission from 1D PCs made of tungsten, which surprisingly, exhibit properties similar to that of the more intricate 3D PC. Such 1D photonic structures have not been investigated as potential candidates for selective emitters for TPV applications.
Thermal radiation in PCs has been treated only indirectly by calculating absorptivity and invoking Kirchoff's law. ${ }^{11}$ Although the framework established long ago is applicable to PCs, ${ }^{12}$ direct simulation has been used infrequently to analyze thermal radiation. ${ }^{13}$ Direct thermal emission modeling has been increasingly used in recent years to study, for example, thermal emission and radiative transfer due to surface waves. ${ }^{14-17}$ Although tedious, direct emission calculation is advantageous because it can also be used to analyze emission from a part of the whole emitter structure. In addition Kirchoff's law can be obtained as a consequence of the direct method. Direct emission calculation is based on determining the dyadic Green's function (GF) ${ }^{18}$ of Maxwell's equations and characterizing the thermally fluctuating current sources using the fluctuation-dissipation (FD) theorem. ${ }^{12}$ We will first present the details of the method. Then we will apply the method to analyze emission from a half-plane and a thin film of silver, and 1D PCs of silver/air and tungsten/dielectric combinations.

The 1D periodic structure we have analyzed is shown in Fig. 1. The structure is a stack of emitters separated by a layer of nonemitting medium, either vacuum or a low-loss dielectric (we consider only nonmagnetic materials). The whole structure is bound between a half-plane of vacuum at one end and a half-plane of nonemitting medium at the other. The Fourier components of the electrical and magnetic fields can be expressed in terms of the dyadic GF as follows:

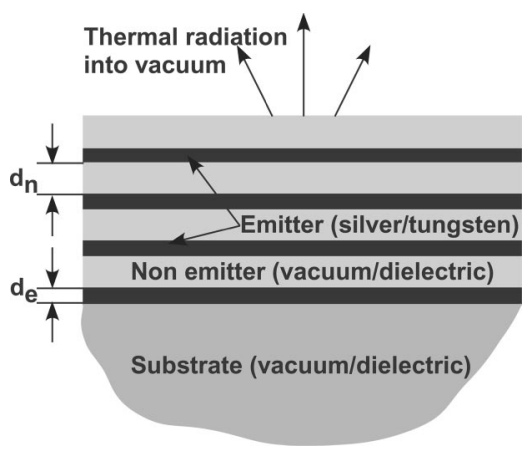

FIG. 1. Schematic of 1D PC. Each unit cell is a combination of emitter and adjacent nonemitter. The structure is bound between a half-plane of vacuum on one side and a substrate (half-plane of lossless dielectric or vacuum) at the other. $d_{e}$ and $d_{n}$ are the thickness of the emitter and nonemitter layers, respectively. 


$$
\begin{gathered}
\mathbf{E}(\mathbf{r} ; \omega)=i \omega \mu_{0} \int \overline{\overline{\mathbf{G}_{e}}}\left(\mathbf{r}, \mathbf{r}^{\prime} ; \omega\right) \cdot \mathbf{J}\left(\mathbf{r}^{\prime} ; \omega\right) d^{3} r^{\prime}, \\
\mathbf{H}(\mathbf{r} ; \omega)=\int \overline{\overline{\mathbf{G}_{h}}}\left(\mathbf{r}, \mathbf{r}^{\prime} ; \omega\right) \cdot \mathbf{J}\left(\mathbf{r}^{\prime} ; \omega\right) d^{3} r^{\prime},
\end{gathered}
$$

where $\overline{\overline{\mathbf{G}_{e}}}\left(\mathbf{r}, \mathbf{r}^{\prime} ; \omega\right)$ and $\overline{\overline{\mathbf{G}_{h}}}\left(\mathbf{r}, \mathbf{r}^{\prime} ; \omega\right)$, the electric and mag-

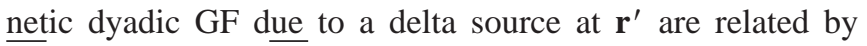
$\overline{\overline{\mathbf{G}_{h}}}\left(\mathbf{r}, \mathbf{r}^{\prime} ; \omega\right)=\nabla_{\mathbf{r}} \times \overline{\overline{\mathbf{G}_{e}}}\left(\mathbf{r}, \mathbf{r}^{\prime} ; \omega\right) ; \mathbf{J}\left(\mathbf{r}^{\prime} ; \omega\right)$ is the Fourier component of the current due to thermal fluctuations; and $\mu_{0}$ is the magnetic permeability of free space. To compute the radiative transfer, we need to determine the Poynting vector at any location. In Cartesian coordinates, the Poynting vector depends on products of the type $E_{i} H_{j}^{*}, E_{j} H_{i}^{*}$, where $i$ and $j$ denote the Cartesian axes and $i \neq j$. Since the source in this case is stochastic, the quantity of interest is $\left\langle E_{i} H_{j}^{*}\right\rangle$, the brackets denote a statistical ensemble averaging. Specifically, the Poynting vector in the $z$ direction depends on the term $\left\langle E_{x} H_{y}^{*}-E_{y} H_{x}^{*}\right\rangle$. Using Eq. (1), we express $\left\langle E_{i} H_{j}^{*}\right\rangle$ as

$$
\begin{aligned}
\left\langle E_{i}\left(\mathbf{r}_{1} ; \omega\right) H_{j}^{*}\left(\mathbf{r}_{1} ; \omega\right)\right\rangle= & i \omega \mu_{0} \int_{V} d^{3} r \int_{V} d^{3} r^{\prime} \\
& \times\left\{G_{e_{i l}}\left(\mathbf{r}_{1}, \mathbf{r} ; \omega\right) G_{h_{m j}}^{T^{*}}\left(\mathbf{r}_{1}, \mathbf{r}^{\prime} ; \omega\right)\right. \\
& \left.\times\left\langle J_{l}(\mathbf{r} ; \omega) J_{m}^{*}\left(\mathbf{r}^{\prime} ; \omega\right)\right\rangle\right\}
\end{aligned}
$$

where $l, m=1,2,3$, and repeated indices refer to summation over all possible combinations; the superscripts $T$ and * refer to transpose and complex conjugate, respectively. To characterize the spectral power density of the thermal source in thermodynamic equilibrium at temperature $T$ we make use of the FD theorem, which expresses the correlations of the microscopic fluctuations of current in terms of a macroscopic parameter, the imaginary part of the dielectric function, as $^{12,14,17}$

$$
\left\langle J_{l}(\mathbf{r} ; \omega) J_{m}^{*}\left(\mathbf{r}^{\prime} ; \omega\right)\right\rangle=\frac{\varepsilon_{0} \varepsilon^{\prime \prime}(\omega) \omega \Theta(\omega, T)}{\pi} \delta_{l m} \delta\left(\mathbf{r}-\mathbf{r}^{\prime}\right),
$$

where $\varepsilon_{0}$ is the electric permittivity of free space; $\varepsilon^{\prime \prime}(\omega)$ is the imaginary part of the dielectric function of the source; and $\Theta(\omega, T)=\hbar \omega /\left(\exp \left(\hbar \omega / k_{B} T\right)-1\right)$, where $2 \pi \hbar$ and $k_{B}$ are Planck's constant and Boltzmann's constant, respectively. With expressions for the dyadic GF and Eq. (3), the expression for $\left\langle E_{i} H_{j}^{*}\right\rangle$ in Eq. (2) can be computed numerically. The problem of determining the radiative transfer is thus converted to a problem of determining the GF for the given case. For the case of layered media, the GF technique has been used extensively. ${ }^{18}$ The GF for layers that do not contain the delta source, which we shall call the homogeneous part, is given by
$\overline{\overline{\mathbf{G}_{e_{l p}}^{H}}}\left(\mathbf{r}, \mathbf{r}^{\prime}\right)=\frac{i}{8 \pi^{2}} \iint \frac{d k_{x} d k_{y}}{k_{z s}}\left\{\begin{array}{c}A_{l} e^{i\left(\mathbf{k}_{l} \mathbf{r}-\mathbf{k}_{s} r^{\prime}\right)} \hat{\mathbf{x}}\left(k_{z l}\right) \hat{\mathbf{x}}\left(k_{z s}\right)+ \\ B_{l} e^{i\left(\mathbf{K}_{l} \cdot \mathbf{r}-\mathbf{k}_{s} \cdot \mathbf{r}^{\prime}\right)} \hat{\mathbf{x}}\left(-k_{z l}\right) \hat{\mathbf{x}}\left(k_{z s}\right)+ \\ C_{l} e^{i\left(\mathbf{k}_{l} \mathbf{r}-\mathbf{K}_{s} \mathbf{r}^{\prime}\right)} \hat{\mathbf{x}}\left(k_{z l}\right) \hat{\mathbf{x}}\left(-k_{z s}\right)+ \\ D_{l} e^{i\left(\mathbf{K}_{l} \cdot \mathbf{r}-\mathbf{K}_{s} \cdot \mathbf{r}^{\prime}\right)} \hat{\mathbf{x}}\left(-k_{z l}\right) \hat{\mathbf{x}}\left(-k_{z s}\right)\end{array}\right\}$,

where $\mathbf{k}_{\|}=k_{x} \hat{\mathbf{e}}_{\mathbf{x}}+k_{y} \hat{\mathbf{e}}_{y}$ is the in-plane wave vector; $\mathbf{k}(\mathbf{K})$ is the wave vector with a component along the $+z(-z)$ direction; $k_{z}=\sqrt{\mathbf{k}^{2}-\mathbf{k}_{\|}^{2}}$ such that $\operatorname{Im}\left(k_{z}\right) \geqslant 0 ; \hat{\mathbf{x}}$ is a unit vector that depends on the polarization of the wave, ${ }^{18}$ subscript $l(s)$ refers to the $l$ th (source) layer; and subscript $p$ refers to the polarization (TE or TM) in consideration. For those layers that contain the $\delta$ source, the GF for a particular polarization $p$ is modified to:

$$
\begin{aligned}
\overline{\overline{\mathbf{G}_{e_{s p}}}}\left(\mathbf{r}, \mathbf{r}^{\prime}\right)= & \overline{\overline{\mathbf{G}_{e_{s p}^{H}}}}\left(\mathbf{r}, \mathbf{r}^{\prime}\right)+\frac{i}{8 \pi^{2}} \iint \frac{d k_{x} d k_{y}}{k_{z s}} \\
& \times\left\{\begin{array}{cc}
e^{i \mathbf{k}_{s} \cdot\left(\mathbf{r}-\mathbf{r}^{\prime}\right)} \hat{\mathbf{x}}\left(k_{z s}\right) \hat{\mathbf{x}}\left(k_{z s}\right) & z>z^{\prime}, \\
e^{i \mathbf{K}_{s} \cdot\left(\mathbf{r}-\mathbf{r}^{\prime}\right)} \hat{\mathbf{x}}\left(-k_{z s}\right) \hat{\mathbf{x}}\left(-k_{z s}\right) & z<z^{\prime},
\end{array}\right.
\end{aligned}
$$

where superscript $H$ indicates that the GF is the homogeneous part; and $\overline{\mathbf{G}_{e_{s p}}^{H}}\left(\mathbf{r}, \mathbf{r}^{\prime}\right)$ is of the form as in Eq. (4). The corresponding magnetic field GF can be determined using the relation between electric and magnetic GF. With the above expressions, the coefficients $A_{l}, B_{l}, C_{l}$, and $D_{l}$ of the GF for a stack of layers bounded by semi-infinite media can be determined using the transfer matrix method. ${ }^{7}$

To analyze thermal emission, the dielectric function of silver is assumed to be $5.17+i-9.013^{2} /(\omega(\omega+i 0.018))$. Below the plasma frequency, thick metal films are generally very good reflectors and bad emitters. As the thickness of the film is reduced to the order of the skin depth, interference effects can increase the emissivity of the films. Figure 2 shows the spectral hemispherical emissivity ${ }^{19}$ of a half-plane of silver as well as a film of thickness $10 \mathrm{~nm}$, both emitting into vacuum. All emissivities have been calculated using the direct emission calculation discussed earlier. We can see from the figure that emissivity of the thin film is higher than that of the half-plane for a significant portion of the frequency spectrum in consideration due to interference effects.

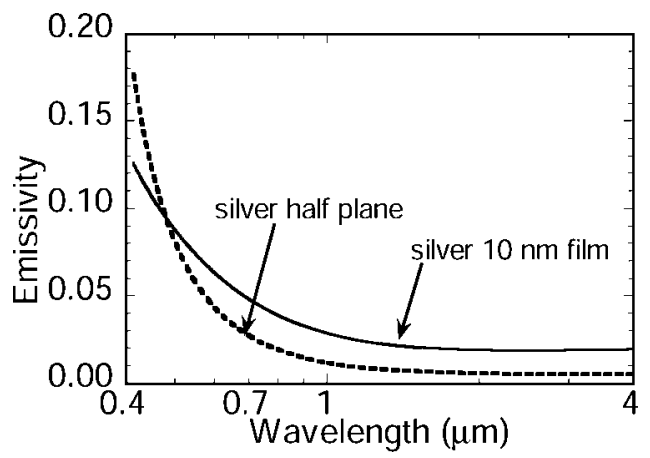

FIG. 2. Spectral hemispherical emissivity of a half-plane and a thin film of silver. 


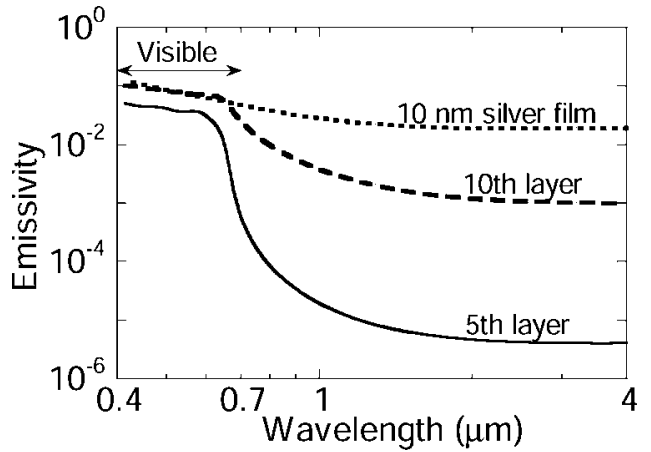

FIG. 3. Spectral hemispherical emissivity of an 11 layer 1D PC stack. The fifth film has five films on either side while the tenth film has one film above and nine films below it.

In order to analyze thermal emission from a PC structure, it would be helpful to understand the effect of the PC structure on the emission from a thin film. That can be done by simply analyzing the emission from only one of the thin films in Fig. 1, without the rest of the structure participating in the emission. In Fig. 3 the results of the analysis of the spectral hemispherical emissivity of a PC of 11 unit cells with $d_{e}=10 \mathrm{~nm}, d_{n}=150 \mathrm{~nm}$, and only one of the thin films participating in the emission are plotted. The rest of the films just act as filters. We see that below a cutoff frequency of about $1.7 \mathrm{eV}(730 \mathrm{~nm})$, the emissivity of the PC structure is reduced by more than two orders of magnitude, even for the emission from a film just one layer below the top-most one. A comparison with the spectral hemispherical emissivity of a bare $10 \mathrm{~nm}$ silver film clearly indicates the effect of the photonic structure. The position of the cutoff frequency can be shifted to higher frequencies by decreasing the vacuum gap between the thin films of silver. Figure 4 shows the spectral hemispherical emissivity of the whole 1D PC with all the thin films acting as emitters as well as filters. It is clear that the effect of the photonic structure is to enhance the emissivity compared to the emissivity of a half-plane of silver, though not equally, in all regions of the spectrum in consideration. This enhancement can be attributed to the top-most emitter. Since the radiation from the top-most emitter observes the effect of the photonic structure to a much lesser extent than the films below, it behaves much the same way a

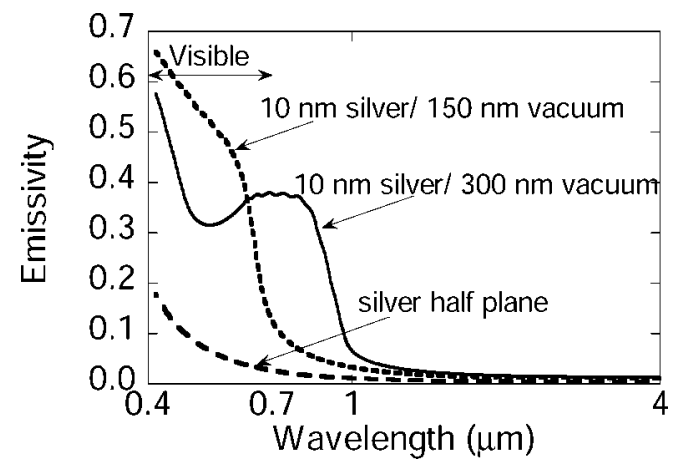

FIG. 4. Spectral hemispherical emissivity of entire PC of 10 unit cells for $10 \mathrm{~nm} \mathrm{Ag}$ films and two values of nonemitter vacuum layer thickness.

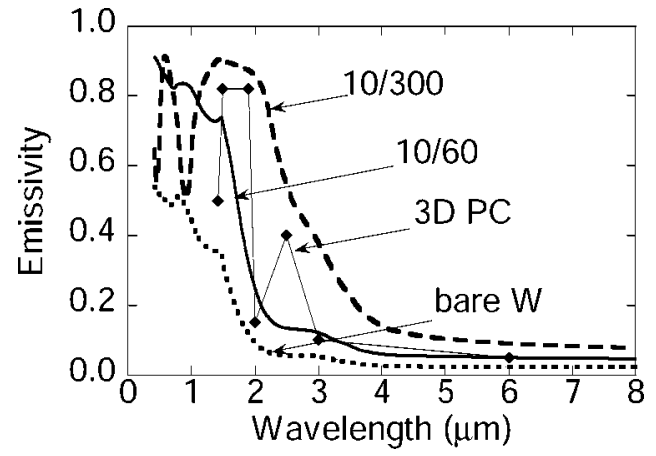

FIG. 5. Emissivity of tungsten/alumina PC. Curves marked $10 / 60$ and $10 / 300$ refer to ID PCs with $10 \mathrm{~nm}$ of tungsten and $60 \mathrm{~nm}$ or $300 \mathrm{~nm}$ of alumina. In both cases, the PCs have 10 unit cells with the metal layer deposited on the substrate. The dots refer to experimental data from a 3D PC (Ref. 2). The lines connecting the dots are only representative of the experimental curve.

solitary thin film of equal size would, resulting in the overall increase as seen in Fig. 1. We also note that by varying the vacuum gap, we could achieve high emissivity for silver in the visible and higher frequencies, while keeping it low in the IR. While silver itself, with its relatively low melting point $(\sim 1213 \mathrm{~K})$, cannot be used for incandescent bulbs, it points towards a simple structure that could potentially greatly improve the luminous efficiency of incandescent light bulbs. Of course, the right material is the key.

Metals are used as emitters for TPV applications because of their inherently low emissivity in the mid- and far-IR. As seen from their usage in incandescent bulbs, tungsten has a relatively good emissivity in the optical (light) as well as near IR (heat) regimes compared to far IR. While the high emissivity in the near IR is a disadvantage for lighting applications, it is very good for TPV applications with low band gap semiconductors like GaSb. In fact, the emissivity of tungsten-based emitters is increased by the appropriate use of antireflective coatings, ${ }^{20} 1 \mathrm{D}$ and 2D surface-relief gratings,, 3 and 3D PCs. ${ }^{2}$ Having seen that 1D PCs can achieve appropriate tailoring of emissivity in the case of silver, we proceed to analyze a similar structure made of tungsten thin films and present another way of tailoring the emissivity. The 1D PC structure itself consists of 10 unit cells, each unit cell composed of a $10 \mathrm{~nm}$ film of tungsten (emitter) and a $60 \mathrm{~nm}$ film of alumina. Instead of assuming a Drude-type dielectric function, as in the case of silver, we have used measured values of optical constants for tungsten from the literature. ${ }^{21}$ We have used a damped oscillator model for the dielectric function of alumina with values taken from the literature. ${ }^{22}$ In order to simulate more closely a practical situation, this PC structure is assumed to be on a dielectric substrate with the metal touching the substrate. The substrate is assumed to have a constant relative electrical permeability of 12 . Although alumina could also act as an emitter, the emission from the alumina layers is negligible $(<1 \%)$ compared to the emission from tungsten. Hence we have plotted only the emission from the tungsten layers.

The spectral hemispherical emissivity of the 1D PC described above is plotted in Fig. 5 along with that for a bare tungsten half-plane and experimental data from the 3D PC. ${ }^{2}$ 
Compared to the emissivity of bare tungsten, the 1D PC shows increased emissivity at all wavelengths in the range considered. In particular, the emissivity in the near IR and optical regimes is around 0.85 while it remains comparatively lower in the far IR regime. By varying the dielectric thickness, the frequency around which the transition from low emissivity $(\sim 0.1)$ to high emissivity $(\sim 0.7)$ occurs can be controlled. Unfortunately the dielectric function of tungsten, unlike that of silver, is such that with a simple 1D PC it seems unlikely that the transition frequency can be decreased below $2.0 \mu \mathrm{m}$. Nonetheless, the 1D PC compares very well with the 3D PC and could be a more practical alternative as an emitter technology for TPV applications.

In summary, the following can be said about thermal emission from 1D MDPCs: (1) Metallic films with thickness of the order of the skin depth, when arranged as a 1D PC, exhibit photonic effects which can be used to tailor the spectral emissivity of the PC structure. Generally, this results in an increase in emissivity in the frequency spectrum in consideration. With the right material, the 1D PC offers a simple structure that could have great implications for incandescent bulb technology. (2) One reason the emissivity increases over a broad spectrum could be the effect of the top-most film. Making structural changes to the top-most film could result in the desired reduction of emissivity. (3) A ID PC structure made of tungsten thin films exhibits enhancements in emissivity remarkably similar to a more complicated 3D PC. The advantage of the ID PC is that it can be fabricated much more easily compared to the 3D PC.

The work is supported by a DOD/ONR MURI (Grant No. N00014-01-1-0803) on Electromagnetic Metamaterials through UCLA.
*Electronic address: gchen2@ mit.edu

${ }^{1}$ J. G. Fleming, S. Y. Lin, I. El-Kady, R. Biswas, and K. M. Ho, Nature (London) 417, 52 (2002).

${ }^{2}$ S. Y. Lin, J. Moreno, and J. G. Fleming, Appl. Phys. Lett. 83, 380 (2003).

${ }^{3}$ A. Heinzel, V. Boerner, A. Gombert, B. Blasi, V. Wittwer, and J. Luther, J. Mod. Opt. 47, 2399 (2000).

${ }^{4}$ M. U. Pralle, N. Moelders, M. P. McNeal, I. Puscasu, A. C. Greenwald, J. T. Daly, E. A. Johnson, T. George, D. S. Choi, I. El-Kady, and R. Biswas, Appl. Phys. Lett. 81, 4685 (2002).

${ }^{5}$ E. R. Brown and O. B. McMahon, Appl. Phys. Lett. 67, 2138 (1995).

${ }^{6}$ D. F. Sievenpiper, E. Yablonovitch, J. N. Winn, S. Fan, P. R. Villeneuve, and J. D. Joannopoulos, Phys. Rev. Lett. 80, 2829 (1998).

${ }^{7}$ O. S. Heavens, Optical Properties of Thin Solid Films (Dover, New York, 1965).

${ }^{8}$ M. Scalora, M. J. Bloemer, A. S. Pethel, J. P. Dowling, C. M. Bowden, and A. S. Manka, J. Appl. Phys. 83, 2377 (1998).

${ }^{9}$ H. Contopanagos, E. Yablonovitch, and N. G. Alexopoulos, J. Opt. Soc. Am. A 16, 2294 (1998).

${ }^{10}$ A. Moroz, Phys. Rev. B 66, 115109 (2002).
${ }^{11}$ C. M. Cornelius and J. P. Dowling, Phys. Rev. A 59, 4736 (1999).

${ }^{12}$ S. M. Rytov, Y. A. Kravtsov, and V. I. Tatarski, Principles of Statistical Radiophysics (Springer, Berlin, 1987), Vol. 3.

${ }^{13}$ L. Tsang, E. Njoku, and J. A. Kong, J. Appl. Phys. 46, 5127 (1975).

${ }^{14}$ R. Carminati and J.-J. Greffet, Phys. Rev. Lett. 82, 1660 (1999).

${ }^{15}$ A. V. Shchegrov, K. Joulain, R. Carminati, and J.-J. Greffet, Phys. Rev. Lett. 85, 1548 (2000).

${ }^{16}$ C. Henkel, K. Joulain, R. Carminati, and J.-J. Greffet, Opt. Commun. 186, 57 (2000).

${ }^{17}$ A. Narayanaswamy and G. Chen, Appl. Phys. Lett. 82, 3544 (2003).

${ }^{18}$ L. Tsang, J. A. Kong, and K. H. Ding, Scattering of Electromagnetic Waves (Wiley, New York, 2000).

${ }^{19}$ M. Quinn Brewster, Thermal Radiative Transfer \& Properties (Wiley-Interscience, New York, 1991).

${ }^{20}$ A. Gombert, AIP Conf. Proc. 653, 123 (2003).

${ }^{21}$ E. D. Palik, Handbook of Optical Constants (Academic, New York, 1985).

${ }^{22}$ M. Gadenne, V. Podolskiy, P. Gadenne, P. Sheng, and V. M. Shalaev, Europhys. Lett. 53, 364 (2001). 